\title{
Caracterização dos óbitos infantis por broncoaspiração, sufocação e síndrome da morte súbita
}

\author{
Characterization of infant deaths by aspiration, suffocation and \\ sudden infant death syndrome
}

\author{
Márcio Souza dos Santos ${ }^{1}$, Patrícia Yoshida ${ }^{2}$, Edilaine Giovanini Rossetto ${ }^{3}$, \\ Rosângela Aparecida Pimenta Ferrari ${ }^{4}$
}

Resumo

\begin{abstract}
Objetivo: descrever e comparar as características relacionadas aos óbitos por causas evitáveis, como a brocoaspiração, sufocação e síndrome da morte súbita, buscando a definição de um perfil comum. Método: trata-se de um estudo de caso, retrospectivo, no qual, foram analisadas as fichas de investigação de óbito infantil, declaração de óbito e declaração de nascido vivo por um período de doze anos. Resultados: dos 944 óbitos ocorridos, 70 (7,4\%) deles tiveram como causa básica de morte a broncoaspiração (45), sufocação (11) ou síndrome da morte súbita (14). O perfil mais frequente encontrado foi: a idade do óbito - pós-neonatal; o local de morte - no domicilio; história de coleito presente; crianças em bom estado de saúde; idade materna entre 20 a 34 anos; baixo nível sócio econômico; fumantes; crianças do sexo masculino; cor branca. Conclusão: reconhecer o perfil desses óbitos irá auxiliar em estratégias de prevenção e redução dos óbitos infantis.
\end{abstract}

Descritores: Mortalidade infantil. Causa básica de morte. Classificação internacional de doenças. Fontes de dados.

\begin{abstract}
Objective: to describe and compare the characteristics related to deaths due to preventable causes, such as brokering, suffocation and sudden death syndrome, seeking a definition of a common profile. Method: this is a retrospective, unqualified case study, being analyzed as records of infant death, declaration of death and declaration of live baby for a period of twelve years. Results: of the 944 deaths, 70 (7.4\%) had basic death due to bronchoaspiration (45), suffocation (11) or sudden death syndrome (14). The most frequent profile was: the age of death - post-neonatal; the place of death - at the address; history of present coleite; children in good health; maternal age between 20 and 34 years; neutron partner; smoking; male children; White color. Conclusion: recognizing the list of auxiliary procedures data on strategies to prevent and reduce infant deaths.

Descriptors: Infant mortality. Underlying cause of death. International classification of diseases. Data sources.
\end{abstract}

\footnotetext{
${ }^{1}$ Residente de Gerência dos Serviços de Enfermagem da Universidade Estadual de Londrina, Londrina, Paraná, Brasil. E-mail: marciosouzaopto@hotmail.com

2 Enfermeira pela Universidade Estadual de Londrina, Londrina, Paraná, Brasil.

3 Doutorado em Enfermagem em Saúde Pública pela Universidade de São Paulo, Ribeirão Preto, São Paulo, Brasil. Professora Adjunto do Departamento de Enfermagem da Universidade Estadual de Londrina, Londrina, Paraná, Brasil.

4 Doutorado em Ciências pela Universidade de São Paulo, São Paulo, São Paulo, Brasil. Professora Adjunta do Departamento de Enfermagem da Universidade Estadual de Londrina. Londrina, Paraná, Brasil.
} 


\section{Introdução}

Em meados da década de 90 foi implantado comitês de vigilância do óbito materno, infantil e fetal que tem por objetivo "[...] compreender as circunstâncias de ocorrência dos óbitos, identificar os fatores de risco e definir políticas de saúde dirigidas a redução da mortalidade materna e infantil. ${ }^{(1)}$ As ações de vigilância do óbito nestes grupos populacionais são divididas em quatro etapas: investigação; análise do óbito; identificação e proposição de medidas preventivas e corretivas relacionadas à assistência e às estatísticas vitais e elaboração de relatórios. Em 2004, o Ministério da Saúde elaborou o Manual dos Comitês de Prevenção do óbito Infantil e Fetal, com propósito de auxiliar e apoiar os locais que não havia ou realizava vigilância do óbito ou comitês municipais e estaduais estruturados.

Os Comitês de Prevenção do Óbito Infantil e Fetal são “[...] organismos interinstitucionais, de caráter eminentemente educativo e formativo, sigiloso [...], (1) tem como proposta nacional aprimorar os registros sobre mortalidade e melhorar a organização da assistência à saúde para a redução de mortes previníveis. $^{(1)} \mathrm{O}$ Comitê Municipal de Prevenção da Mortalidade Materna e Infantil (CMPMMI) está vigente desde 1.999 no município de Londrina, e a partir de 2004 analisa $100 \%$ dos óbitos maternos e infantil contribuindo para melhoria da qualidade de informação, maior fidedignidade de correção de causa básica no Sistema de Informação sobre Mortalidade (SIM).

A causa básica de morte entre menores de 1 ano de idade, segundo a Fundação Sistema de Análise de Dados (SEADE), classifica os óbitos em: evitáveis, não evitáveis, mal definidos e não classificados. No que se refere aos óbitos classificados como evitáveis identifica-se redutíveis por: imunoprevenção; adequado controle na gravidez; adequada atenção ao parto; ações de prevenção, diagnóstico e tratamento precoce; e através de parceria com outros setores. Ressalta-se que a classificação das causas evitáveis seguem a Classificação Internacional de Doenças e Problemas Relacionadas à Saúde (CID-10). ${ }^{(1)}$

O presente estudo trata de uma análise de óbitos de crianças menores de um ano em que a causa básica de óbito foram sufocação, broncoaspiração e síndrome da morte súbita, que se configuram em óbitos ou mortes redutíveis através de parcerias com outros setores, especificamente as causas externas capítulo XX da CID-10.

Sufocação (W75) significa o impedimento da passagem de ar por meio direto, oclusão das vias respiratórias, ou indireto, compressão do tórax e abdome impedindo os movimentos respiratórios. (2) Broncoaspiração (W78.0-78.5) é definido como a aspiração de conteúdo gástrico para a árvore traqueobrônquica. Sufocação e broncoaspiração de acordo com a (CID-10), estão inseridos no (Capítulo XX Causas externa de morbidade e de mortalidade), no grupo (W75-W84), categoria (W75) e (78.0-78.5) respectivamente. ${ }^{(3)}$ Síndrome da morte súbita além de possuir causa multifatorial é caracterizada pela morte repentina e abrupta, ocorrida durante o sono, que permanece sem explicação após investigação e necropsia do corpo. (4) Segundo a (CID-10), está contida no (Capítulo XVIII Sintomas, sinais e achados anormais de exames clínicos e de laboratório, não classificados em outra parte), no grupo (R95-R99 Causas mal definidas e desconhecidas de mortalidade). ${ }^{(3)}$

O presente estudo tem por objetivo descrever e comparar as características relacionadas a esses óbitos, buscando a definição de um perfil comum. Para que possa futuramente auxiliar em estratégias de prevenção e redução dos óbitos infantis por essas causas básicas de morte.

\section{Método}

Trata-se de um estudo de caso, exploratório e retrospectivo. O estudo de caso se destaca por " [...] sua característica de estudar uma unidade, bem delimitada e contextualizada, com a preocupação 
de não analisar apenas o caso em si, como algo à parte, mas o que ele representa dentro do todo e a partir daí. ${ }^{(5)} \mathrm{O}$ delineamento do estudo de caso como metodologia de investigação é dividido em quatro fases: delimitação da unidade caso; coleta de dados; seleção, análise e interpretação de dados e; elaboração do relatório do caso. ${ }^{(5)}$ As aplicações são variadas tendo como destaque pesquisas exploratórias e comparativas.

O estudo foi realizado no município de Londrina segunda cidade mais populosa do Estado do Paraná, localizado na região norte do Paraná, com uma população total de 563.943 habitantes, sendo que 6.935 são menores de 1 ano. ${ }^{(6)}$

A população de estudo foi constituída de crianças menores de um ano, residentes em Londrina, que faleceram entre 2000 e 2012 cujas causas básicas de morte foram: broncoaspiraçã, sufocação e síndrome da morte súbita na infância segundo a (CID-10). Do total de 944 óbitos infantis ocorridos em Londrina de 2000 e 2012, foram incluídos na pesquisa 70 $(7,4 \%)$ casos de óbito, deles tiveram como causa básica de morte a broncoaspiração (45), sufocação (11) ou síndrome da morte súbita (14). ${ }^{(7)}$

As variáveis estudadas foram agrupadas em três blocos, o primeiro está contido as características do óbito, local de atendimento pós-óbito e codificação pré e pós investigação do óbito. No segundo incluíram-se as características maternas, reprodutivas, obstétricas e econômicas, e no último as características da criança e pós-nascimento.

Foi utilizada como fonte de dados a Declaração de Nascido Vivo (DN) obtidos a partir do Sistema de Informações sobre Nascidos Vivos (SINASC), Declarações de Óbito (DO) e a Ficha de Investigação de Óbito Infantil oriundas do Comitê Municipal de Prevenção de Mortalidade Materna e Infantil (CMPMMI), arquivadas no Núcleo de Informação sobre Mortalidade (NIM) da Secretaria de Saúde do Município.

Por ser uma pesquisa envolvendo seres humanos, mesmo que indiretamente pela utilização de dados secundários (DO, DN, Ficha de Investigação de Óbito Infantil), foram seguidos os procedimentos éticos, estabelecidos na Resolução Número 196, de 10 de outubro de 1996, do Conselho Nacional de Saúde, que dispõe sobre Diretrizes e Normas.

O estudo foi submetido ao Comitê de Ética em Pesquisa da Universidade Estadual de Londrina - CEP/UEL, tendo sido aprovado sob o número: 843/2009/CEPEEUSP, em 02 de setembro de 2009, CAAE 0044.0.196.00009/843/2009.

\section{Resultados e Discussão}

No período de 2000 e 2012 ocorreram 70 óbitos envolvendo as causas: broncoaspiração, sufocação e síndrome da morte súbita. Por se tratar de óbitos de difícil determinação causal, devem-se ser consideradas algumas limitações: dados secundários; erros no preenchimento da DO; erro de preenchimento e de dados disponíveis (DO, DN, Ficha de investigação de óbito infantil), conhecimento e treinamento inadequado por parte dos profissionais em realizar a autopsia verbal; necessidade da necropsia e de profissionais com treinamento especifico para o diagnóstico após a morte e possibilidade de subdiagnóstico.

Conforme apresenta a tabela 1 os óbitos por essas causas específicas ocorreram em sua maioria no período pós-neonatal principalmente para o grupo R95-99. Estudo realizado em Pelotas aponta maior prevalência em crianças com idade inferior a 1 mês. ${ }^{(8)}$

Quanto à estação do ano se mostrou um fator comum entre broncoaspiração e sufocação, prevalecendo o inverno como um fator mais frequente para ambos. O local em que ocorreu oóbito, no domicilio, demonstrou ser uma característica predominante para todas as causas básicas estudadas no presente estudo, para as três causas. Dormir junto com os pais no inverno, se apresentou novamente como esse fator frequentemente presente de óbito por sufocação e broncoaspiração, pois é neste 
período que se usa mais agasalhos e cobertas e o coleito torna-se frequente devido ao frio. ${ }^{(9)}$

A prevalência do coleito no mundo varia significativamente, de país para país, no Brasil são escassos estudos realizados em torno desse tema. Estudo feito em Pelotas demonstrou que a maioria (70\%) tinham a prática da realização do coleito. ${ }^{(8)}$ Neste estudo não foi observado associação entre coleito e mãe sem companheiro. O presente estudo não foi observado relação entre coleito e mãe sem companheiro.

A modificação da causa básica se mostrou como um fator revelador, dos 70 óbitos estudados, 21 tiveram alterações em suas causas básicas de morte, o que demonstra várias falhas durante o processo da codificação da causa de morte. Estudo aponta que para que se atinja níveis satisfatórios de qualidade dos dados sobre causas de morte, é imprescindível um correto e completo preenchimento da Declaração de Óbito, desta forma possibilita $=$ que as ações dos serviços de saúde estejam em consonância com as necessidades das populações. ${ }^{(10)}$ A fim de se reduzir DO emitidas com causa básica de morte mal definida, utiliza-se a ficha de Investigação de Óbito Infantil, onde é coletado dados de vários serviços. Quando esses dados se apresentam insuficientes para se esclarecer a causa básica de morte, é utilizado o método Autópsia Verbal (AV), é coletado informações sobre sinais e sintomas apresentados anteriormente ao óbito, com a família e cuidadores, diante disto é possível destaca-se a importância dos dados completos para que haja a investigação do óbito de forma a esclarecer os óbitos. ${ }^{(11-12)}$

Por serem óbitos contidos em um universo de difícil determinação causal e 88,57\% terem ocorrido no domicilio, fora do serviço de saúde, seria preciso a realização da necropsia nestes casos. A necropsia é um fator determinante no diagnóstico de óbito de difícil determinação causal, contribui para um diagnóstico de causa básica mais assertivo. Ela é somente obrigatória nos casos de mortes violentas ou não naturais, mas quando há necessidade de estabelecer com precisão a causa básica ela deverá ser realizada. Do total de óbitos apenas 38 realizaram necropsia, evidenciando que muitos dos óbitos podem ter sido declarados, causa básica de morte, erroneamente. O Serviço de Vigilância de Óbito vem também ajudar a elucidar a causa básica de morte, pois cabe a ele "[...] buscar informações junto aos familiares, utilizar a documentação disponível sobre o acompanhamento clinico do falecido, usar o método da AV e também valer-se de necropsia, a fim de estabelecer a causa do óbito". (11-12) 
Tabela 1 - Caracterização dos óbitos infantis por causas mal definidas e desconhecidas de mortalidade (R95-99), broncoaspiração (W78.0-78.5) e sufocação (W75.0) de acordo com a análise do contexto do óbito, 2000 a 2012, Londrina-PR.

\begin{tabular}{|c|c|c|c|c|c|c|c|}
\hline \multirow{2}{*}{ DESCRIÇÃO } & & \multicolumn{2}{|c|}{ R95-99 } & \multicolumn{2}{|c|}{ W78.0-78.5 } & \multicolumn{2}{|c|}{ W75.0 } \\
\hline & & $\mathbf{N}$ & $\%$ & $\mathbf{N}$ & $\%$ & $\mathbf{N}$ & $\%$ \\
\hline \multicolumn{8}{|c|}{ CARACTERÍSTICAS DO ÓBITO } \\
\hline \multirow{3}{*}{$\begin{array}{l}\text { Classificação do } \\
\text { óbito em dias }\end{array}$} & Neonatal precoce & 0 & 0,0 & 1 & 2,2 & 0 & 0,0 \\
\hline & Neonatal tardio & 1 & 7,1 & 4 & 8,8 & 4 & 36,4 \\
\hline & Pós-neonatal & 13 & 92,8 & 40 & 88,8 & 7 & 63,6 \\
\hline \multirow{4}{*}{ Estação do ano } & Verão & 3 & 21,4 & 7 & 15,6 & 2 & 18,2 \\
\hline & Outono & 5 & 35,7 & 13 & 28,9 & 1 & 9,1 \\
\hline & Inverno & 1 & 7,1 & 16 & 35,5 & 5 & 45,4 \\
\hline & Primavera & 5 & 35,7 & 9 & 20,0 & 3 & 27,3 \\
\hline \multirow{6}{*}{ Local do óbito } & Hospital terciário & 1 & 7,1 & 2 & 4,4 & 1 & 9,1 \\
\hline & Domicilio & 12 & 85,7 & 40 & 88,9 & 10 & 90,9 \\
\hline & Creche & 0 & 0,0 & 1 & 2,2 & 0 & 0,0 \\
\hline & Via pública & 1 & 7,1 & 0 & 0,0 & 0 & 0,0 \\
\hline & Outra cidade & 0 & 0,0 & 1 & 2,2 & 0 & 0,0 \\
\hline & Centro de Apoio à Recuperação Infantil & 0 & 0,0 & 1 & 2,2 & 0 & 0,0 \\
\hline \multirow{3}{*}{$\begin{array}{l}\text { Dormia junto com } \\
\text { os pais }\end{array}$} & Sim & 3 & 21,4 & 21 & 46,7 & 7 & 63,6 \\
\hline & Não & 1 & 7,1 & 4 & 8,9 & 3 & 27,3 \\
\hline & Não informado & 10 & 71,4 & 20 & 44,4 & 1 & 9,1 \\
\hline \multicolumn{8}{|c|}{ LOCAL DE ATENDIMENTO PÓS ÓBITO } \\
\hline \multirow{3}{*}{$\begin{array}{l}\text { Criança em bom } \\
\text { estado de saúde } \\
\text { antes do óbito }\end{array}$} & $\operatorname{Sim}$ & 12 & 85,7 & 39 & 86,7 & 11 & 100,0 \\
\hline & Não & 2 & 14,3 & 5 & 11,1 & 0 & 0,0 \\
\hline & Não identificado & 0 & 0,0 & 1 & 2,2 & 0 & 0,0 \\
\hline \multirow{3}{*}{$\begin{array}{l}\text { Número de servi- } \\
\text { ços utilizados pós } \\
\text { óbito }\end{array}$} & Nenhum & 9 & 64,3 & 8 & 17,8 & 4 & 36,4 \\
\hline & 1 & 5 & 35,7 & 29 & 64,4 & 6 & 54,5 \\
\hline & 2 & 0 & 0,0 & 8 & 17,8 & 1 & 9,1 \\
\hline \multirow{3}{*}{$\begin{array}{l}\text { Serviços de Saú- } \\
\text { de utilizados pós } \\
\text { óbito }\end{array}$} & SAMU/SIATE & 1 & 7,14 & 21 & 46,7 & 3 & 27,3 \\
\hline & Unidade Básica de Saúde & 2 & 14,3 & 6 & 13,3 & 0 & 0,0 \\
\hline & Hospital & 2 & 14,3 & 13 & 28,9 & 5 & 45,5 \\
\hline \multicolumn{8}{|c|}{ CODIFICAÇÃO PRÉ E PÓS ÓBITO } \\
\hline \multirow{2}{*}{$\begin{array}{l}\text { Modificação } \\
\text { da causa básica }\end{array}$} & Sim & 3 & 21,4 & 13 & 28,9 & 5 & 45,5 \\
\hline & Não & 11 & 78,6 & 32 & 71,1 & 6 & 54,5 \\
\hline \multirow[t]{2}{*}{ Necropsia } & Sim & 4 & 28,6 & 28 & 62,2 & 6 & 54,5 \\
\hline & Não & 10 & 71,4 & 17 & 37,8 & 5 & 45,5 \\
\hline
\end{tabular}

Fonte: Autores.

Ao analisar a tabela 2, as características maternas, tiveram fator comum em todos os óbitos: idade entre 20 a 34 anos; escolarização inferior a 8 anos; ter companheiro; fumante e não exercer ocupação fora do lar. No grupo R95-99 resultados foram similares com outro estudo, uma vez que os óbitos por síndrome da morte súbita tinham como características: mães fumantes, a idade materna entre 20 e 23 anos. $^{(8)}$ 
Tabela 2 - Caracterização dos óbitos infantis por causas mal definidas e desconhecidas de mortalidade (R95-99), broncoaspiração (W78.0-78.5) e sufocação (W75.0) de acordo com as características socioeconômicas, maternas, reprodutivas e obstétrica, 2000 a 2012, Londrina, PR.

\begin{tabular}{|c|c|c|c|c|c|c|c|}
\hline \multirow[t]{2}{*}{ DESCRIÇÃO } & & \multicolumn{2}{|c|}{ R95-99 } & \multicolumn{2}{|c|}{ W78.0-78.5 } & \multicolumn{2}{|c|}{ W75.0 } \\
\hline & & $\mathbf{N}$ & $\%$ & $\mathbf{N}$ & $\%$ & $\mathbf{N}$ & $\%$ \\
\hline \multicolumn{8}{|c|}{ CARACTERÍSTICAS MATERNAS } \\
\hline Idade em anos & $\begin{array}{l}<20 \\
20-34 \\
>=35\end{array}$ & $\begin{array}{l}6 \\
8 \\
0\end{array}$ & $\begin{array}{c}42,9 \\
57,1 \\
0,0\end{array}$ & $\begin{array}{c}15 \\
26 \\
4\end{array}$ & $\begin{array}{c}33,3 \\
57,8 \\
8,9\end{array}$ & $\begin{array}{l}2 \\
8 \\
1\end{array}$ & $\begin{array}{c}18,2 \\
72,7 \\
9,1\end{array}$ \\
\hline $\begin{array}{l}\text { Escolaridade } \\
\text { em anos }\end{array}$ & $\begin{array}{l}<8 \\
8 \text { e mais } \\
\text { Nenhuma } \\
\text { Não informado }\end{array}$ & $\begin{array}{l}7 \\
6 \\
0 \\
1\end{array}$ & $\begin{array}{c}50,0 \\
42,9 \\
0,0 \\
7,1\end{array}$ & $\begin{array}{c}30 \\
13 \\
1 \\
1\end{array}$ & $\begin{array}{c}66,7 \\
28,9 \\
2,2 \\
2,2\end{array}$ & $\begin{array}{l}7 \\
4 \\
0 \\
0\end{array}$ & $\begin{array}{r}63,6 \\
36,4 \\
0,0 \\
0,0\end{array}$ \\
\hline Situação conjugal & $\begin{array}{l}\text { Com companheiro } \\
\text { Sem companheiro } \\
\text { Não informado }\end{array}$ & $\begin{array}{l}8 \\
6 \\
0\end{array}$ & $\begin{array}{c}57,1 \\
42,9 \\
0,0\end{array}$ & $\begin{array}{c}27 \\
16 \\
2\end{array}$ & $\begin{array}{c}60,0 \\
35,6 \\
4,4\end{array}$ & $\begin{array}{l}7 \\
3 \\
1\end{array}$ & $\begin{array}{r}63,6 \\
27,3 \\
9,1\end{array}$ \\
\hline Fumante & $\begin{array}{l}\text { Sim } \\
\text { Não } \\
\text { Não informado }\end{array}$ & $\begin{array}{l}7 \\
7 \\
0\end{array}$ & $\begin{array}{c}50,0 \\
50,0 \\
0,0\end{array}$ & $\begin{array}{c}19 \\
20 \\
6\end{array}$ & $\begin{array}{l}42,2 \\
44,4 \\
13,3\end{array}$ & $\begin{array}{l}8 \\
2 \\
1\end{array}$ & $\begin{array}{c}72,7 \\
18,2 \\
9,1\end{array}$ \\
\hline Trabalha fora & $\begin{array}{l}\text { Sim } \\
\text { Não } \\
\text { Não identificado }\end{array}$ & $\begin{array}{c}1 \\
13 \\
0\end{array}$ & $\begin{array}{c}7,1 \\
92,9 \\
0,0\end{array}$ & $\begin{array}{c}11 \\
32 \\
2\end{array}$ & $\begin{array}{c}24,4 \\
71,1 \\
4,4\end{array}$ & $\begin{array}{l}4 \\
7 \\
0\end{array}$ & $\begin{array}{r}36,4 \\
63,6 \\
0,0\end{array}$ \\
\hline Renda familiar em salário & $\begin{array}{l}<1 \\
1 \text { a } 2,9 \\
3 \text { e mais } \\
\text { Não informado }\end{array}$ & $\begin{array}{l}5 \\
8 \\
0 \\
1\end{array}$ & $\begin{array}{c}35,7 \\
57,1 \\
0,0 \\
7,1\end{array}$ & $\begin{array}{c}6 \\
19 \\
5 \\
15\end{array}$ & $\begin{array}{l}13,4 \\
42,2 \\
11,1 \\
33,3\end{array}$ & $\begin{array}{l}1 \\
4 \\
2 \\
4\end{array}$ & $\begin{array}{c}9,1 \\
36,4 \\
18,2 \\
36,4\end{array}$ \\
\hline $\begin{array}{l}\text { Número de residentes na } \\
\text { casa }\end{array}$ & $\begin{array}{l}2-4 \\
>=5 \\
\text { Não informado }\end{array}$ & $\begin{array}{l}6 \\
4 \\
1\end{array}$ & $\begin{array}{c}42,8 \\
28,6 \\
7,1\end{array}$ & $\begin{array}{l}11 \\
13 \\
12\end{array}$ & $\begin{array}{l}24,5 \\
28,9 \\
26,7\end{array}$ & $\begin{array}{l}4 \\
4 \\
2\end{array}$ & $\begin{array}{l}36,4 \\
36,4 \\
18,2\end{array}$ \\
\hline
\end{tabular}

Fonte: Autores.

Fumar e a prática de coleito são visto como um fator de risco para síndrome da morte súbita em vários estudos, a Academia Americana de Pediatria recomenda que pais que dividem a cama com a criança não devem fumar e nem usar substâncias alcoólicas e ilícitas, observa-se no entanto que os famíliares não relacionam a prática com riscos para a vida de seus filhos. ${ }^{(8-9)}$

As características sócio econômicas evidenciam que os óbitos pertenciam à classe econômica desfavorecida. Estudos demonstram que o baixo nível sócio-econômico é um fator de risco para todos as causas de mortalidade infantil..$^{(7,13,14)} \mathrm{Um}$ estudo de caso controle realizado em Maceió, analisou os fatores de risco para mortalidade neonatal, observou-se como um fator determinante residências com menor número de moradores e com ausência de filhos menores de um ano. ${ }^{(13)} \mathrm{O}$ número de residentes não se mostrou como um comum entre as causas de óbitos. 
Tabela 3 - Caracterização dos óbitos infantis por causas mal definidas e desconhecidas de mortalidade (R95-99), broncoaspiração (W78.0-78.5) e sufocação (W75.0) de acordo com as características maternas reprodutivas e obstétrica ocorridas, 2000 a 2012, Londrina, PR.

\begin{tabular}{|c|c|c|c|c|c|c|c|}
\hline \multirow[t]{2}{*}{ DESCRIÇÃO } & & \multicolumn{2}{|c|}{ R95-99 } & \multicolumn{2}{|c|}{ W78.0-78.5 } & \multicolumn{2}{|c|}{ W75.0 } \\
\hline & & $\mathbf{N}$ & $\%$ & $\mathbf{N}$ & $\%$ & $\mathbf{N}$ & $\%$ \\
\hline \multicolumn{8}{|c|}{ CARACTERÍSTICAS REPRODUTORAS E OBSTÉTRICAS MATERNAS } \\
\hline Número de gestações & $\begin{array}{l}\text { Primípara } \\
\text { Multípara }\end{array}$ & $\begin{array}{l}6 \\
8\end{array}$ & $\begin{array}{l}42,9 \\
57,1\end{array}$ & $\begin{array}{l}14 \\
31\end{array}$ & $\begin{array}{l}31,1 \\
68,9\end{array}$ & $\begin{array}{l}2 \\
9\end{array}$ & $\begin{array}{l}18,2 \\
81,8\end{array}$ \\
\hline Realizou o pré natal & $\begin{array}{l}\text { Sim } \\
\text { Não }\end{array}$ & $\begin{array}{c}13 \\
1\end{array}$ & $\begin{array}{c}92,9 \\
7,1\end{array}$ & $\begin{array}{c}40 \\
5\end{array}$ & $\begin{array}{l}88,9 \\
11,1\end{array}$ & $\begin{array}{c}10 \\
1\end{array}$ & $\begin{array}{c}90,9 \\
9,1\end{array}$ \\
\hline $\begin{array}{l}\text { Número de consultas de } \\
\text { pré natal }\end{array}$ & $\begin{array}{l}\text { Nenhuma } \\
1 \text { a } 6 \\
7 \text { a } 10 \\
10 \text { e mais } \\
\text { Não informado }\end{array}$ & $\begin{array}{l}1 \\
6 \\
7 \\
0 \\
0\end{array}$ & $\begin{array}{c}7,1 \\
42,9 \\
50,0 \\
0,0 \\
0,0\end{array}$ & $\begin{array}{c}5 \\
18 \\
10 \\
1 \\
1\end{array}$ & $\begin{array}{c}11,1 \\
40,0 \\
44,4 \\
2,2 \\
2,2\end{array}$ & $\begin{array}{l}1 \\
6 \\
4 \\
0 \\
0\end{array}$ & $\begin{array}{c}9,1 \\
54,5 \\
36,4 \\
0,0 \\
0,0\end{array}$ \\
\hline Pré natal de risco & $\begin{array}{l}\text { Sim } \\
\text { Não } \\
\text { Não identificado } \\
\text { Não realizou pré-natal }\end{array}$ & $\begin{array}{c}2 \\
10 \\
1 \\
1 \\
\end{array}$ & $\begin{array}{c}14,3 \\
71,4 \\
7,1 \\
7,1 \\
\end{array}$ & $\begin{array}{c}11 \\
21 \\
8 \\
5\end{array}$ & $\begin{array}{l}24,5 \\
46,7 \\
17,8 \\
11,1\end{array}$ & $\begin{array}{l}0 \\
9 \\
1 \\
1 \\
\end{array}$ & $\begin{array}{c}0,0 \\
81,9 \\
9,1 \\
9,1 \\
\end{array}$ \\
\hline
\end{tabular}

Fonte: Autores.

As características obstétricas e reprodutivas maternas se revelaram em comum para todos os óbitos. O número de mães multíparas entre os óbitos por sufocação foi de $81,8 \%$ seguido por $68,9 \%$ broncoaspiração e $57,1 \%$ causas mal definidas e desconhecidas. Mães multíparas neste estudo foi considerada um fator comum, outro estudo aponta o inverso, o número de mães multíparas foi inferior a primíparas. ${ }^{(14)}$

A assistência no pré-natal foi considerada adequada em termos de número de consultas e a realização do pré-natal. Resultados similares foram encontrados em um estudo quando este aponta uma cobertura elevada da assistência pré-natal $(98,7 \%)$ tendo $75,8 \%$ das mulheres iniciado o pré-natal antes da $16^{\mathrm{a}}$ semana gestacional e $73,1 \%$ compareceram a seis ou mais consultas. ${ }^{(14)}$

De acordo com a tabela 4 no que diz respeito ao sexo, predominou o masculino em todas causas básicas (78,6\%). Vários estudos sobre síndrome da morte súbita têm obtido resultados idênticos sobre a prevalência do sexo masculino nesta causa de morte, tornando um fator de risco. ${ }^{(13)}$ Quanto à etnia, a raça branca teve maior proporção em todas as causas básica de morte. ${ }^{(8)}$

Com relação às características de nascimento, verifica-se que a maioria dos casos ocorreram em crianças nascidas com idade gestacional 37 a 41 semanas e peso $>2500 \mathrm{~g}$, estando adequados para nascimento. Mas, observou-se também a ocorrência de nascimentos prematuros, aproximadamente $30 \%$. Quase a totalidade das crianças nasceu sem asfixia (Apgar $>7$ ).

O tipo de parto predominante realizado pelas mães foi o vaginal. Mas, o parto cesáreo representou $28,57 \%$ do total de óbitos. O estudo realizado pela PNDS demonstrou que o percentual de partos cesárea no Brasil é muito alto 43,8\%, a Organização Mundial da Saúde (OMS) recomenda no máximo $15 \%$ desse tipo de parto.

A média de internação pós-nascimento entre as causas básica de morte, foi de $41,42 \%$, porém este resultado não se apresenta fidedigno, pois $20 \%$ dos óbitos não tinham dados sobre internação nos registros. Um estudo de caso controle realizado 
em Maceió (AL) buscou identificar os fatores de risco para mortalidade neonatal, e teve como fator determinante à internação de recém-nascido na UTI. ${ }^{(14)}$

Em relação à vacinação atualizada, $90,0 \%$ dos óbitos por sufocação estavam em dia com a vacina, seguido de $80,0 \%$ por broncoaspiração e $71,4 \%$ por causas mal definidas e desconhecidas. Estudos comprovam que a vacina é um fator de proteção para mortalidade infantil, mas não há na literatura, pesquisas atestando que a imunização oferece ou não proteção para essas.

Tabela 4 - Caracterização dos óbitos infantis por causas mal definidas e desconhecidas de mortalidade (R95-99), broncoaspiração (W78.0-78.5) e sufocação (W75.0) de acordo com as características da criança, 2000 a 2012, Londrina, PR

\begin{tabular}{|c|c|c|c|c|c|c|c|}
\hline \multirow{2}{*}{ DESCRIÇÃO } & & \multicolumn{2}{|c|}{ R95-99 } & \multicolumn{2}{|c|}{ W78.0-78.5 } & \multicolumn{2}{|c|}{ W75.0 } \\
\hline & & $\mathbf{N}$ & $\%$ & $\mathbf{N}$ & $\%$ & $\mathbf{N}$ & $\%$ \\
\hline \multicolumn{8}{|c|}{ CARACTERISTICAS DA CRIANÇA } \\
\hline Gemelaridade & $\begin{array}{l}\text { Sim } \\
\text { Não }\end{array}$ & $\begin{array}{c}1 \\
13\end{array}$ & $\begin{array}{l}7,1 \\
92,8\end{array}$ & $\begin{array}{c}6 \\
39\end{array}$ & $\begin{array}{l}13,3 \\
86,7\end{array}$ & $\begin{array}{c}1 \\
10\end{array}$ & $\begin{array}{c}9,1 \\
90,9\end{array}$ \\
\hline Sexo & $\begin{array}{l}\text { Feminino } \\
\text { Masculino }\end{array}$ & $\begin{array}{c}3 \\
11\end{array}$ & $\begin{array}{l}21,4 \\
78,5\end{array}$ & $\begin{array}{l}18 \\
27\end{array}$ & $\begin{array}{l}40,0 \\
60,0\end{array}$ & $\begin{array}{l}3 \\
8\end{array}$ & $\begin{array}{l}27,3 \\
72,7\end{array}$ \\
\hline Raça/cor & $\begin{array}{l}\text { Branca } \\
\text { Parda } \\
\text { Preta } \\
\text { Indígena }\end{array}$ & $\begin{array}{c}11 \\
1 \\
2 \\
0\end{array}$ & $\begin{array}{c}78,6 \\
7,1 \\
14,3 \\
0,0\end{array}$ & $\begin{array}{c}32 \\
11 \\
1 \\
1\end{array}$ & $\begin{array}{c}71,1 \\
24,4 \\
2,2 \\
2,2\end{array}$ & $\begin{array}{l}7 \\
4 \\
0 \\
0\end{array}$ & $\begin{array}{c}63,6 \\
36,4 \\
0,0 \\
0,0\end{array}$ \\
\hline \multicolumn{8}{|c|}{ CARACTERÍSTICAS PÓS NASCIMENTO } \\
\hline $\begin{array}{l}\text { Idade gestacional no nasci- } \\
\text { mento em semanas }\end{array}$ & $\begin{array}{l}<31 \\
32 \text { a36 } \\
37 \text { a } 42\end{array}$ & $\begin{array}{l}0 \\
5 \\
9\end{array}$ & $\begin{array}{c}0,0 \\
35,7 \\
64,3\end{array}$ & $\begin{array}{c}7 \\
8 \\
30\end{array}$ & $\begin{array}{l}15,6 \\
17,8 \\
66,7\end{array}$ & $\begin{array}{l}1 \\
3 \\
7\end{array}$ & $\begin{array}{c}9,1 \\
27,3 \\
63,6\end{array}$ \\
\hline Peso ao nascer em gramas & $\begin{array}{l}<1.000 \\
1.000 \text { a } 2.499 \\
2.500 \text { e mais } \\
\text { Não identificado }\end{array}$ & $\begin{array}{l}0 \\
6 \\
9 \\
0\end{array}$ & $\begin{array}{c}0,0 \\
42,9 \\
57,1 \\
0,0\end{array}$ & $\begin{array}{c}3 \\
13 \\
29 \\
0\end{array}$ & $\begin{array}{c}6,7 \\
28,9 \\
64,4 \\
0,0\end{array}$ & $\begin{array}{l}0 \\
3 \\
7 \\
1\end{array}$ & $\begin{array}{c}0,0 \\
27,3 \\
63,6 \\
9,1\end{array}$ \\
\hline Apgar no $5^{\circ}$ minuto & $\begin{array}{l}4 \text { a } 6 \\
7 \text { a } 10 \\
\text { Não informado }\end{array}$ & $\begin{array}{c}0 \\
14 \\
0\end{array}$ & $\begin{array}{c}0,0 \\
100,0 \\
0,0\end{array}$ & $\begin{array}{c}1 \\
40 \\
4\end{array}$ & $\begin{array}{c}2,2 \\
88,9 \\
8,9\end{array}$ & $\begin{array}{l}0 \\
9 \\
2\end{array}$ & $\begin{array}{c}0,0 \\
81,8 \\
18,2\end{array}$ \\
\hline Tipo de parto & $\begin{array}{l}\text { Cesárea } \\
\text { Vaginal }\end{array}$ & $\begin{array}{l}5 \\
9\end{array}$ & $\begin{array}{l}35,7 \\
64,3\end{array}$ & $\begin{array}{l}14 \\
31\end{array}$ & $\begin{array}{l}31,1 \\
68,9\end{array}$ & $\begin{array}{c}1 \\
10\end{array}$ & $\begin{array}{c}9,1 \\
90,9\end{array}$ \\
\hline Internação após o nascimento & $\begin{array}{l}\text { Sim } \\
\text { Não } \\
\text { Não informado }\end{array}$ & $\begin{array}{l}6 \\
7 \\
1\end{array}$ & $\begin{array}{c}42,9 \\
50,0 \\
7,1\end{array}$ & $\begin{array}{l}19 \\
14 \\
12\end{array}$ & $\begin{array}{l}42,2 \\
31,1 \\
26,7\end{array}$ & $\begin{array}{l}4 \\
6 \\
1\end{array}$ & $\begin{array}{c}36,4 \\
54,5 \\
9,1\end{array}$ \\
\hline Vacinação atualizada & $\begin{array}{l}\text { Sim } \\
\text { Não } \\
\text { Não informado }\end{array}$ & $\begin{array}{l}10 \\
3 \\
1\end{array}$ & $\begin{array}{c}71,4 \\
21,4 \\
7,1\end{array}$ & $\begin{array}{c}36 \\
3 \\
6\end{array}$ & $\begin{array}{c}80,0 \\
6,7 \\
13,3\end{array}$ & $\begin{array}{c}10 \\
0 \\
1\end{array}$ & $\begin{array}{c}90,0 \\
0,0 \\
9,1\end{array}$ \\
\hline Aleitamento & $\begin{array}{l}\text { Aleitamento materno exclusivo } \\
\text { Aleitamento materno } \\
\text { Não Amamenta } \\
\text { Não informado }\end{array}$ & $\begin{array}{l}5 \\
3 \\
5 \\
1\end{array}$ & $\begin{array}{c}35,7 \\
21,4 \\
35,7 \\
7,1\end{array}$ & $\begin{array}{c}21 \\
10 \\
10 \\
4\end{array}$ & $\begin{array}{c}46,7 \\
22,2 \\
22,2 \\
8,9\end{array}$ & $\begin{array}{l}7 \\
4 \\
0 \\
0\end{array}$ & $\begin{array}{c}63,6 \\
36,4 \\
0,0 \\
0,0\end{array}$ \\
\hline
\end{tabular}

Fonte: Autores. 
O tipo de aleitamento que prevaleceu entre as causas básicas de morte estudadas foi o aleitamento materno exclusivo, a causa por sufocação obteve o maior valor de $63,6 \%$. O mesmo ocorre em outro estudo, em que apresenta que $62 \%$ de aleitamento materno exclusivo. ${ }^{(8)}$

\section{Conclusão}

As causas básicas de morte analisadas neste estudo são de difícil determinação causal, evidenciado pelo número de casos que tiveram a causa básica modificado, ou seja, é preciso uma investigação mais aprofundada e além de tudo conhecimento e comprometimento dos profissionais responsáveis pelo preenchimento da DO.

O cenário dos óbitos estudados ocorreu de modo semelhante para as três causas básicas de morte estudadas. Os fatores predominantes associados nestes óbitos foram: idade do óbito no período pósneonatal; o local de morte - no domicilio; história de coleito presente; crianças em bom estado de saúde; idade materna entre 20 a 34 anos; baixo nível sócio econômico; fumantes; criança do sexo masculino, cor branca, parto vaginal e vacinação atualizada.

Por meio deste estudo observou-se a importância na qualidade dos dados registrados e o impacto na produção da informação, portanto, é preciso uma capacitação específica para investigação mais assertiva nestes casos, além do conhecimento e comprometimento dos profissionais envolvidos. Sugere-se a adoção da investigação de óbito por causa mal definida e a utilização do formulário de autópsia verbal proposto pelo Ministério da Saúde para também ser aplicado sistematicamente nestes casos, uma vez que a elucidação dos fatores frequentemente presentes nesses óbitos poderá auxiliar em estratégias de prevenção e redução dos óbitos infantis por essas causas básicas de morte.

\section{Referências}

1 Ministério da Saúde (BR). Secretaria de Vigilância em Saúde. Secretaria de Atenção à Saúde. Manual de vigilância do óbito infantil e fetal e do Comitê de Prevenção do Óbito Infantil e Fetal. [Internet] Brasília: Ministério da Saúde; 2009 [citado 2019 abr. 4]. Disponível em: http://bvsms.saude.gov.br/bvs/ publicacoes/manual_obito_infantil_fetal_2ed.pdf.

2 França GV. Medicina Legal. 9a. ed. Rio de Janeiro (RJ): Guanabara Koogan; 2011.

3 Organização Mundial da Saúde [OMS]. Classificação Internacional de Doenças e problemas relacionados à saúde: décima revisão (CID-10). São Paulo: Centro Colaborador da OMS para Classificação de Doenças em Português; 2008.

4 Martins MEP, Amorim LM, Rodrigues MN, Lima DGS, Moreira JFM. Síndrome da morte súbita infantil (smsi): aspectos acerca das principais causas e as formas de prevenção. Id on Line Rev. Mult. Psci. 2018; 12 (41): 192-205.

5 Ribas JLC. Relato de caso e seu potencial na pesquisa. Rev Saúde Desenvolv. 2017; 11(7):5-9.

6 Instituto Paranaense de Desenvolvimento Econômico e Social [IPARDES]. Caderno Estatístico [Internet]. Paraná (PR): Instituto Paranaense de Desenvolvimento Econômico e Social; 2018 [citado 2018 jun. 20]. Disponível em: http://www.ipardes.gov.br/cadernos/ MontaCadPdf1.php?Municipio=19\&btOk=ok.

7 Londrina. Prefeitura Municipal. Caderno Estatístico de Londrina. [Internet]. Londrina (PR): Prefeitura Municipal; 2018 [citado 2018 jun 20]. Disponível em: http://www.ipardes.gov.br/cadernos/MontaCadPdf1. php?Municipio $=86000$.

8 Lorea RL, Pilger MC, Ceia ML. Síndrome da morte súbita infantil em Pelotas de 2006 a 2013: uma análise descritiva. Rev Med. 2017; 96(1): 27-31.

9 Pankiw PM, Landowsky TF, Godoi VC, Klossowski DG, Rodrigues AH, Fujinaga CI. Dormir compartilhado e a morte súbita no contexto de famílias fumicultoras. Rev Univ Vale do Rio Verde. 2016; 14(2): 994-1000.

10 Carolina CC, Renato T, Elisabeth F. Avaliação da investigação de óbitos por causas mal definidas no Brasil em 2010. Epidemiol. Serv. Saude. 2017; 26(1):19-30.

11 Bittencourt DAS, Dias MAB, Wakimoto MD. Vigilância do óbito, infantil e fetal e atuação em comitês de mortalidade. Rio de Janeiro: EDA/Ensp; 2013. 
12 Santos SPC, Lansky S, Ishitani LH, Franca EB. Óbitos infantis evitáveis em Belo Horizonte: análise de concordância da causa básica, 2010-2011. Rev. Bras. Saúde Matern. Infant. 2015; 15(4): 389-99.

13 Rodrigues LS, Melo SF, Melo EVCF, Araújo A. Aspectos importantes sobre a mortalidade infantil em Itapecerica - Minas Gerais. Rev. Enferm. Cent.-Oeste Min. 2013; 3(1): 498-506.

14 Viellas EF, Domingues RMSM, Dias MAB, Gama SGN, Theme Filha MM, Costa JV, et al. Assistência pré-natal no Brasil. Cad. Saúde Pública. 2014; 30(1): S85-S100. Doi: 10.1590/0102-311X00126013 